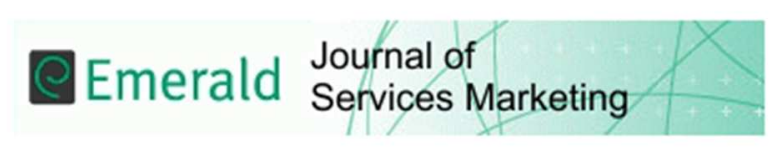

\title{
Information Richness and Trust in V-Commerce: Implications for Services Marketing
}

\begin{tabular}{|r|l|}
\hline Journal: & Journal of Services Marketing \\
\hline Manuscript ID & JSM-02-2015-0099.R3 \\
\hline Manuscript Type: & Article \\
\hline Keywords: & $\begin{array}{l}\text { Information richness, Trust, e-commerce and v-commerce, virtual worlds, } \\
\text { experimental design }\end{array}$ \\
\hline \multicolumn{2}{|l}{} \\
\hline
\end{tabular}

SCHOLARONE

Manuscripts 


\title{
Information Richness and Trust in V-Commerce: Implications for Services Marketing
}

\begin{abstract}
Purpose: The potential for e-commerce is limited by a trust deficit when traders do not interact in a physical, bricks-and-mortar context. The theory of information richness posits that equivocal interactions, such as ones requiring trust, can be facilitated through communication media that transmit multiple cues interactively. We examine the potential of information-rich virtual worlds to reduce this trust deficit compared with more traditional web-based e-tailing environments.

Design/Methodology: Rather than focusing on stated intentions we adopt an experimental approach to measure behaviour. Participants receive performance-related financial incentives to perform trust games in different information-rich treatments that represent three retail environments: a physical environment representing bricks-and-mortar trade, an electronic environment representing web-based online retailing and a virtual environment representing virtual world retail.
\end{abstract}

Findings: We find that the two dimensions of trust significantly differ between the treatments. In particular, as hypothesised, both trustingness and trustworthiness are higher in the virtual than in the electronic environment. However, contrary to our hypotheses, physical trade is not associated with greater trust than virtual trade.

Implications: We extend previous research by demonstrating how the information richness of the virtual world interface can promote e-commerce by deepening trust between trading partners. Our research also complements existing work that approaches product and service interfaces through the lens of servicescapes. The findings also contribute towards the development of services marketing practice and the design of e-commerce environments.

Keywords: information richness, trust, e-commerce and v-commerce, virtual worlds, experimental design 


\section{Introduction}

Electronic commerce (e-commerce) has transformed how business is conducted, how business partners interact and how information is shared between them (Agarwal \& Wu, 2015). Different types of e-commerce platform abound. Among them, virtual worlds have been called 'a significant new market environment for brand-building through experiential customer service interactions' (Barnes, Mattsson, \& Hartley, 2015, p. 12) and their impact on service innovation has been noted (Barrett, Davidson, Prabhu, \& Vargo, 2015). Despite their promise, electronic trading environments bear risks in the much greater scope for anonymity. The associated signals that moderate trade in traditional environments are not present in electronic shopping environments. As a result, online shopping is characterised by the lack of trust (Bauer, Albrecht, Neumann, \& Haber, 2015, p. 57) and consequently a greater element of risk (Quigley, 2015, p. 7) which may deter consumers. To reduce consumers' inherent distrust of websites, firms need to develop ways to overcome the lack of physical cues that consumers use to assess quality, safety or security (Beatty, Reay, Dick, \& Miller, 2011, p. 2).

Trust is significant in electronic marketplaces (Bansal, Zahedi, \& Gefen, 2016) especially as social capital in online activities (Schlichter \& Rose, 2013). In the service literature, the role of trust is especially highlighted in the importance of brand credibility and the impact of strong service on increasing consumer trust (Berry, 2000). However, research in this area is still lacking (Bansal et al., 2016 , p. 1). The key question for online retailers is how to retain existing customers and provide repeat purchase opportunities (C. Johnson, Hult, \& McGowan, 2008). Ostrom et al. (2015) posited thirteen priorities for the service industry, one of which is the need to leverage technology to advance service. V-commerce provides the key to such leverage through building trust as part of online commerce activities. Technology in the v-commerce space may also provide the key to marrying the benefits of bricks-and-mortar spaces while minimising the inherent trust barriers present in ecommerce. This marriage may also shed light on commitment-trust theory and responds to calls for work considering service contexts to refine these foundational theories and to build service knowledge (Ostrom et al., 2015).

These observations raise the issue of how electronic retail spaces should be designed to minimise loss of trust compared to physical retail environments. In the current paper, we address this issue through an experiment based on the theory of information richness which objectively categorises different communication media according to their capacity to carry information and promote shared meaning and understanding when ambiguity exists (Daft, 2013; Rockmann \& 
Northcraft, 2008). The theory predicts that information richer electronic media such as virtual environments engender greater trust between interaction partners compared to less rich ones such as web interfaces. However, they produce lesser trust compared to face-to-face interactions. We test this notion using an online experiment with participants playing an economic game designed to measure trust in relation to monetary incentives via different media. We find that virtual worlds can be highly effective in instilling trust, which we attribute to their relative information richness. The main contribution of this work is to extend previous research by demonstrating how the information richness of the virtual world interface can promote e-commerce by deepening trust between trading partners. Our research also complements existing work that approaches product and service interfaces through the lens of servicescapes. The findings also contribute towards the development of services marketing practice and the design of e-commerce environments.

The remainder of this paper is organised as follows. The next section contains our literature review. We discuss the construct of trust, its significance in the context of electronically-mediated commerce, the theoretical framework we use to examine the relationship between the two and our research questions. The subsequent section outlines our research approach and design. Results are presented next followed by conclusions, including a discussion of findings, limitations, and implications.

\section{Trust, E-commerce and Information Richness}

\section{Conceptualising Trust}

Recent literature has highlighted the key role of trust in commercial interactions generally and in retail trade specifically (Toufaily, Souiden, \& Ladhari, 2013). Mutual trust between trading partners is an important lubricant of trade (Gefen \& Straub, 2004) whenever asymmetric information creates a potential for opportunism that can only be checked at the transaction cost of monitoring and enforcement (San Martín \& Camarero, 2005). At its most general, trust refers to a person's expectation that interaction partners will act in a cooperative manner based on shared norms. However, beneath this surface, trust is a multifaceted concept (Waytz, Heafner, \& Epley, 2014, p. 113) with multiple definitions and measurements (Grabner-Kräuter \& Bitter, 2013; Hupcey, Penrod, Morse, \& Mitcham, 2001). One's trust can relate specifically to a particular person and how it develops over the relationship with that person (Mutz, 2005). Alternatively, it can relate to other people generally, such as strangers (Gefen, Benbasat, \& Pavlou, 2008). Trust applies to 'conditions of uncertainty and vulnerability' regarding how much one person puts at risk in an interaction 
(trustingness) or how one repays the risk one's partner has invested (trustworthiness) (Kelton, Fleischmann, \& Wallace, 2008, p. 365). In psychological terms, trust may be conceptualised as a belief regarding other people (cognitive trust), as an emotional response to reliance on others, as an intention or disposition to trust, or in terms of an individual's actual actions (Gefen \& Straub, 2004; Komiak \& Benbasat, 2004; Mayer, Davis, \& Schoorman, 1995; McKnight, Choudhury, \& Kacmar, 2003). As a result a plethora of individual-level measurement scales of trust exist depending on the context of the interaction (Butler, 1991) including integrity (Gefen \& Straub, 2004; Giffin, 1967), predictability (Gefen \& Straub, 2004; McKnight et al., 2003; Rotter, 1971), ability (Dwyer, Schurr, \& Oh, 1987; Gefen \& Silver, 1999; Gefen \& Straub, 2004), benevolence (Blau, 1964; Gefen \& Straub, 2004; Giffin, 1967; Luhmann, 1979), dependability (Rotter, 1980), honesty (Kumar, 1996) and competence (McKnight et al., 2003). The most common questionnaire-based measurement of trust at the individual level is generalised trust towards strangers, which may be aggregated over groups or nations to generate indices (e.g. Inglehart, 1997).

\section{Measuring Trust}

While many studies use questionnaires to measure individuals' attitudes and intentions to trust others and to return their trust (Gefen \& Straub, 2004; Inglehart, 1997) there are problems with this approach. A host of studies have demonstrated weak or non-existent correlations between different trust survey instruments and participants' observed trust behaviour (Glaeser, Laibson, Scheinkman, \& Soutter, 2000; Holm \& Danielson, 2005). This state of affairs reflects plentiful general evidence from social psychology that questionnaire responses, whether they regard attitudes or intended behaviour, may significantly diverge from actual behaviour (see Chandon, Morwitz, \& Reinartz, 2005 for an overview). In particular, there is doubt concerning the validity and reliability of survey measures for a number of reasons: First, survey measures are not incentive compatible, i.e. when a particular survey response is not linked to resulting financial consequence and respondents may, therefore, exercise little mental effort or veracity in answering the question (Verhallen \& Pieters, 1984). Second, cognitive factors such as the wording and ordering of the questions, as well as the types of scales presented have been shown to affect the way respondents answer the questions. Third, cognitive dissonance may cause respondents to record answers to achieve consistency with their past attitudes and behaviours. Finally, respondents are known to underreport socially undesirable values and attitudes perhaps due to self-serving biases or strategic considerations. Selfreported intention-to-trust variables, commonly used as proxies for trust, may suffer from the above weaknesses (Bertrand \& Mullainathan, 2001).

While these observations suggest merit in measuring trust - by observing behaviour rather 
than through attitudinal responses, such an approach poses practical difficulties. These consist of observing relevant instances of trusting behaviour in participants such as the sharing of personal information, making a purchase or acting on information received (McKnight et al., 2003, p. 337). Even if possible, observational research of this type may be prohibitively costly (Grabner-Kräuter \& Kaluscha, 2003, p. 803). An alternative to the collection of data in naturalistic settings comes from experimental research. Here, incentivised individual behaviour is observed in laboratory settings using abstract tasks designed to elicit the behaviour of interest while controlling confounding influences (see Roth (1988) for an overview). In particular, the trust game (Berg, Dickhaut, \& McCabe, 1995) is commonly used to observe behaviours relating to trust and trustworthiness. In this two-player sequential game, a 'sender' chooses any part of a stake of money to send to a 'receiver'. Whatever part is sent is tripled before the receiver decides how much of it to return to the sender. The sender's payoff is whatever he or she has not sent plus what is received back; the receiver gets whatever he or she has not returned to the sender. The trust game provides measurements of the two dimensions of trust (Camerer \& Fehr, 2004). The percentage of the stake the sender sends to the receiver measures trustingness, i.e. the risk placed in the interaction partner. The percentage of the received sum the receiver returns measures trustworthiness, the degree to which a person repays the trustingness placed in them by the interaction partner.

In theory, a money-maximising receiver would return nothing. In anticipation, a moneymaximising sender would send nothing in the first place. In contrast, both players' payoffs are collectively maximal if the sender sends the entire stake before being tripled, and if the receiver returns half of it. This socially more efficient outcome can be attributed to trustingness and trustworthiness respectively, i.e. the fulfilled expectation by the sender that the receiver will return more than the amount sent. The average amount sent in trust game experiments is typically about half of the stake. Receivers tend to return the absolute amount sent to them, i.e. about a third of the tripled amount (e.g. Bolle, 1998; Croson \& Buchan, 1999; Willinger, Keser, Lohmann, \& Usunier, 2003). In the 15 years following its introduction there have been over 150 experimental studies using the standard trust game (N. D. Johnson \& Mislin, 2011). This large literature has established a host of factors that affect trustingness and trustworthiness in experiments. These include attitudes toward risk, demographics (culture, gender and age) as well as structural variables in the experiment such as anonymity (see Camerer (2003) for an overview).

\section{Trust and Physical Interactions}

Trust has particular significance in the context of e-commerce. Bricks-and-mortar retail spaces and physical trade benefit from established formal and informal arrangements and the presence of 
identifiable partners that reduce transaction cost and risks associated with informational deficiencies. Traditional, but static, electronic retail environments have much greater scope for anonymity as they do not involve physical interactions or signals that moderate trade in traditional environments. In offline spaces, the object of trust is typically a person or entity (firm). However, in an online context, the technology (primarily the Internet) and the firm deploying that technology (sellers and the companies they represent) are the objects of trust (Beldad, De Jong, \& Steehouder, 2010; Doney \& Cannon, 1997). Customers and prospects in electronic commerce have to trust not only the website but also the company behind the website (Doney \& Cannon, 1997). The greater degree of uncertainty that characterises electronic transactions makes them particularly prone to low trust (Grabner-Kräuter \& Kaluscha, 2003) due to the relative lack of formal (procedures and regulations) and informal (conventions and customs) arrangements between e-traders, and lesser ability to monitor and enforce compliance (for example, inspecting products and service delivery) (Gefen \& Straub, 2004). The result is a greater potential for opportunism in others which necessitates costly negotiating, monitoring and enforcing compliance (Fang et al., 2014). Electronic environments also offer scope for deception, such as lying about gender (e.g. Castelfranchi \& Tan, 2001).

Further, the typical lack of interpersonal and repeated interactions in the relatively anonymous electronic trading environments tends to reduce the potential for trust. In particular, ecommerce lacks two major aspects of physical co-presence that engender trust between interaction partners. First, physicality raises the awareness of the co-presence of another sentient being accompanied by a sense of engagement with the other (Biocca, Burgoon, Harms, \& Stoner, 2001). Secondly, the other's physical presence produces human physiological and psychological mechanisms that can promote pro-social behaviour patterns, including trust. Physical presence allows for non-verbal communication, including a host of involuntary signals such as voice tone, body language, pupil dilation, blushing and perspiration which reveal emotional states that provide information about the other person. For instance, eye contact, touching and proximity are used to signal conversational intimacy, agreement and liking (Argyle \& Dean, 1965). Conversely, the relative anonymity and absence of interaction partners in electronic trading environments result in a lack of physiological signals and body language that may lead to significantly reducing trust between them (e.g. Bente, Ruggenberg, Kramer, \& Eschenburg, 2008; Cyr, Hassanein, Head, \& Ivanov, 2007; Gefen \& Straub, 2004; Shin \& Shin, 2011). Overall, the flipside of the promise that ecommerce holds is the potential for a trust deficit. In the words of Francis Fukuyama:

trust does not reside in integrated fibre optic cables [...] A "virtual" firm can have abundant information coming through network wires about its 
suppliers and contractors. But if they are all crooks or frauds, dealing with them will remain a costly process involving complex contracts and time consuming enforcement (Fukuyama, 1995, p. 25).

Following the above arguments, we hypothesise that both dimensions of trust would be greater in interactions taking place in physical environments, compared to electronic ones, as follows:

H1: Trustingness is greater in physical environments compared to electronic environments.

$\mathrm{H} 2$ : Trustworthiness is greater in physical environments compared to electronic environments.

\section{Trust and Information Richness}

The issue of low trust in e-commerce is important as both the nature and significance of e-commerce as a part of the overall economy are continuously developing. There is a growing need for marketing researchers and practitioners to design novel information technology mechanisms to establish trust when individuals trade via electronic interfaces (Fang et al., 2014). Such research can consult a vast literature within computer-mediated communication (CMC) studies that deals with medium design and choice. It contains multiple theoretical frameworks including various forms of presence (copresence, telepresence, social presence, see Nowak and Biocca 2003 ), immersion (Blascovich et al., 2002), vividness (Coyle \& Thorson, 2001), channel expansion and information richness (Daft, 2013). Theories of this kind typically classify different media according to the extent to which their features simulate various aspects of face-to-face interaction (Postmes, Spears, Sakhel, \& de Groot, 2001). One popular example is the theory of information richness which posits that a medium's capacity to reduce ambiguity in interactions lies in its ability to transmit rich information, which in turn depends on its objective features (Carlson \& Zmud, 1999; Daft, 2013; El-Shinnawy \& Markus, 1998; Kock, 2004; Rockmann \& Northcraft, 2008; Simon \& Peppas, 2004). The information richness a particular medium achieves is determined by four criteria (Daft, Lengel, \& Trevino, 1987, p. 358). First, feedback is the degree to which interaction partners can respond to one another instantaneously. Second, multiple cues is the extent to which the message can be accompanied by non-verbal communication or signals from the other's physical presence or social identity. Third, language variety relates to the range of transmittable symbols such as natural language as well as numerical or graphical information. Fourth, personal focus is the extent to which personal (motivations, feelings, perceptions) and situational characteristics can be part of the interaction. Different media are classified hierarchically regarding their ability to transmit rich information through their particular features. Face-to-face is the richest medium followed by telephone, written addressed (e-mail, SMS) 
and finally unaddressed documents such as websites.

More information-rich media and communication channels are therefore better at handling ambiguous interactions, i.e. situations where the amount of facts and data is not enough for a decision (El-Shinnawy \& Markus, 1998). Ambiguity (or equivocality) is a common feature in business (Daft \& Lengel, 1986) whereby there are conflicting interpretations and interests that undermine mutual understanding which cannot be solved by more information but rather require experience and judgment. Trust in e-commerce is an example of such ambiguous interactions (Rockmann \& Northcraft, 2008, p. 109). As we have seen, an individual's decision to trust is associated with risk to the extent that interests between partners are not aligned, and the transaction cost of checking opportunism are prohibitively high. Trust decisions cannot be made merely through analysis and entail both cognitive and emotional responses (Rockmann \& Northcraft, 2008) to engage the decision maker's experience and intuition.

When interactions involving trust are mediated electronically, ambiguity is further raised through the absence of physical cues. Conversely, interactions conducted via richer media should, therefore, be associated with greater trust between interaction partners (Rockmann \& Northcraft, 2008, p. 109) and offer greater scope for combining the benefits of electronic interfaces with the information richness of real, physical retail settings. In ambiguous situations, the richness rather than the amount of information matters to the decision maker. In support, Daft and Lengel (1987) find business managers tend to choose more information rich media when dealing with equivocal and illdefined situations. Other studies found that evaluations of e-tailing websites are positively related to their information richness (Coyle \& Thorson, 2001; Simon \& Peppas, 2004).

We may use information richness theory to classify two of the main e-commerce platforms we consider here: websites and virtual worlds. According to the objective criteria of the theory (Carlson \& Zmud, 1999; Daft, 2013; Kock, 2004), websites have lower information richness than virtual worlds. When online shoppers use retail websites there is typically no instant feedback or interaction with retailer representatives. Cues and language are impoverished in that they consist solely of unaddressed text and images which, according to Daft and Lengel (1987, p. 359), from the lowest level of richness due to lack of personal focus. On the other hand, individual websites can differ in information richness depending on their particular features (Lu, Kim, Dou, \& Kumar, 2014). While still electronic, virtual world retail spaces are commonly designed to transmit richer cues than websites. Retail assistants are often present in the form of avatars with which shoppers can communicate using real-time text chat or audio telephony. This virtual interaction, therefore, allows for personal focus and multiple cues such as the ones transmitted by avatar appearance, gestures and movement. 
Trust in V-commerce

The discussion so far suggests that information-rich media such as virtual worlds may provide an approach to overcoming the potential for a trust deficit associated with electronic commerce. Virtual worlds combine the benefits of the electronic trading environment with the information richness of face-to-face trading through features that compensate for the loss of physical presence associated with e-commerce. Virtual world commerce (v-commerce) is a form of e-commerce where commercial transactions are conducted inside a virtual world. A virtual world is a persistent, threedimensional computer-based environment where many networked users interact using avatars as their graphical representations (Figures 1 and 2). Interactions include aspects of social life such as pregnancy and birth (Lomanowska \& Guitton, 2014), education and learning (Noteborn, DaileyHebert, Carbonell, \& Gijselaers, 2014) even the development and understanding of palaeontology (Cunningham, Rahman, Lautenschlager, Rayfield, \& Donoghue, 2014).

While virtual worlds began as a platform for gaming they have developed into a more general mode of social and commercial interaction. Virtual world activity is now not only socially (Bainbridge, 2007), but also commercially, significant (Castronova, 2008; Knowles, Castronova, \& Ross, 2015a; Prentice, 2007). In virtual worlds, people interact in fully functioning economies based on convertible currencies and the potential for significant returns (Bainbridge, 2007). In such worlds, 'users collect, trade, destroy, produce, and refine virtual resources' just as they would in real worlds (Knowles et al., 2015a). As a result, 3D social virtual worlds have become the 'mainstream Internet application' based on over ten years development in the USA (Zhang et al., 2014, p. 578).

One of the leading virtual worlds to have developed beyond the game-playing origins of this medium is Second Life (SL). As of early 2009, SL has over 15 million registered accounts, and by April 2013, this number had more than doubled to 33 million user accounts (Linden Research 2013). Unlike multi-player, role-playing games such as World of Warcraft, SL has no stipulated goal associated with participation and is purely an online social environment (Greiner, Caravella, \& Roth, 2014). SL is open-ended and, therefore, resists classification; its users employ it in a variety of ways including role play, traditional games, social networking and commerce. SL can best be seen as an arena of creativity, with examples of common activities ranging from sport and recreation (e.g. fishing, skydiving) to getting married and raising virtual children. The interface allows for the creation of multifarious objects that can be programmed with behaviour that enables users to accomplish pretty much anything they can imagine.

SL is unusual among virtual worlds in that the intellectual property of any object belongs to its creator. The interface also allows for the immediate and costless transfer of money between users in the form of the Linden dollar (L\$) which is fully convertible at a floating, but relatively stable, 
exchange rate with the U.S. dollar. SL facilitates v-commerce because any product can be designed and built by one user, then sold and delivered to another. Also, physical objects can also be sold for delivery outside of SL and vice versa. While not yet on the same scale as web-facilitated commerce, $\mathrm{v}$-commerce through mediums such as SL is becoming increasingly significant (for a summary of the economic decision-making and marketplace characteristics of SL see Duffy (2011)). A typical SL shopping scene can be seen in Figure 1.

Due to their large user base and increasingly developed and vigorous labour, product and service markets (Chambers, 2011), virtual worlds are fast becoming important phenomena for social science research (Knowles, Castronova, \& Ross, 2015b). However, existing work regarding the potential of virtual environments in promoting trust is still limited. This paper is intended as a contribution towards this.

As discussed above, electronic environments can be classified in terms of their information richness. Environments richer in information are expected to engender higher levels of trust. Websites and virtual worlds lie on lower and higher ends of the media richness spectrum respectively. As such, in terms of the dimensions of trust, we hypothesise as follows:

H3: Trustingness is greater in virtual-based electronic environments compared to website-based ones. H4: Trustworthiness is greater in virtual-based electronic environments compared to website-based ones.

\section{Virtual Trust Experiments}

Virtual worlds may combine some of the most desirable aspects of physical trading with the benefits of electronic commerce. Such a finding could generate new perspectives in marketing and selling as well as in the broader context of changing business interactions (Greiner et al., 2014). As such, this topic merits further research and investigation. In the current paper we examine the role of vcommerce in instilling trust in online retailing using the trust game experimental approach. The purpose of this paper is to use a trust game experiment to assess whether virtual worlds (as an alternative to e-tail websites) can promote trust between traders in electronic environments. Our objective is to examine whether the two different facets of trust are more prevalent in the virtual context compared with trade in web-based e-commerce settings.

There are a number of reasons in favour of such an approach. As we have seen, existing research suggests that experiments with trust games can generate behavioural measurements of trust that address issues associated with questionnaire research on trust. Further, this approach measures generalised trust in anonymous interactions and not longitudinally, which arguably captures the 
nature of most computer-mediated transactions (Gefen et al., 2008, p. 227; Gefen \& Straub, 2004, p. 410). Another reason is that trust games have been previously successfully used to assess the effect of information richness on trust behaviour. For example, Füllbrunn et al. (2011) compare trust games played in SL with ones conducted in a physical laboratory. Atlas and Putterman (2011) compare trust games in two different SL settings manipulated to vary richness using virtual visual cues. There is also one other study that used trust game experiments in studying e-commerce trust (Keser, 2002) which focuses on the effect reputation management systems, such as published customer ratings of sellers, have on trust. The finding is that both forms of trust game behaviour are enhanced in the experimental treatment representing the reputation management system.

\section{Research Method and Design}

We have seen that the further progress of e-commerce may be hindered by its potential for low trust between trading partners when information richness is low compared with physical retail. Also, we argued that one promising avenue to overcome this problem lies in information-rich virtual worlds, electronic trading environments that simulate certain aspects of physical and social settings. We now outline how we examined this possibility in our experiment. We first outline our main variables, their measurement, and our treatments. We then discuss the research design including operationalising ecommerce and v-commerce in our experiment to test whether differences in trust game behaviour exist between the two.

\section{Measures}

Our main research question is whether v-commerce may be a platform to alleviate some of the trust issues associated with e-commerce conducted on less information-rich website environments. In particular, our specific objective is to assess whether trading in virtual environments is characterised by greater degrees of trust. We follow the experimental literature and measure trust as the amount sent by the sender (trustingness) and returned by the receiver (trustworthiness) in a standard, financially incentivised trust game. Further, to vary information richness, our trust games were conducted in three environments of objectively differing information richness, including a websitebased one representing the e-commerce environment (EE), a virtual world one representing vCommerce (VE). We also create a control treatment in a physical laboratory representing physical trade in traditional settings (PE). In the information richness hierarchy, $\mathrm{PE}$ is associated with greater richness than the $\mathrm{CMC}$-environments $\mathrm{EE}$ and $\mathrm{VE}$. In turn, due to its features, $\mathrm{VE}$ is more information 
rich than EE. Following the information richness theory, we propose that trust is positively associated with the information richness of the environment within which the two parties interact. Pursuant to our hypotheses, we expect that trustingness and trustworthiness is greater in PE compared to EE or VE and that they are greater in VE compared to EE.

\section{Experimental Design}

Our study follows standard experimental economics methodology (Croson, 2005) and involves incentive-compatible tasks, i.e. where the elicited behaviour determines the participant's financial reward. This work builds on previous projects examining behaviour inside virtual worlds experimentally (Chesney, Chuah, \& Hoffmann, 2009). Participants were recruited to play incentivised trust games in three different treatments, corresponding with the PE, EE and VE settings described above. In each, participants played in separate groups for senders and receivers respectively. They remained anonymous to each other and were randomly selected throughout. All participants were briefed using written instructions (available upon request) and had to complete a comprehension quiz successfully before being permitted to indicate their decisions. We now discuss how the experimental protocol differed between the three treatments.

\section{Implementation}

The PE-setting of the experiment was implemented as follows. First, a total of 96 participants, i.e. 48 sender-receiver pairs were recruited via standard e-mail solicitation from the participant database of the Centre for Decision Research and Experimental Economics at the University of Nottingham, UK. A total of seven scheduled sessions were conducted in a physical laboratory and, in each, participants were randomly divided into two groups, senders and receivers. Participants were told that their coparticipant would be randomly chosen from the other group but would otherwise remain anonymous. Thus, there was no interaction between participants. Participants received written instructions but were also briefed orally on the rules of the trust game. Each participant had to complete a quiz before making decisions to ensure comprehension. The task commenced when all participants correctly completed the quiz. One form was used to record participant identification numbers and the amounts sent, received and returned. The forms first were distributed among senders and collected after decisions were indicated. Subsequently, the forms were shuffled and randomly distributed to receivers to indicate their decisions. Payoffs resulting from both participants' decisions were paid out in cash at the end of each session. Each participant also received a show-up fee of $£ 5$ (U. S. \$7.50). In the sessions, participants played the standard trust game as described before with the following parameter values: senders had to choose any part of a stake of $£ 4$ (U.S. \$ 6), in multiples of $£ 0.50$ 
(USD $\$ 0.75$ ) to be sent to the receiver. The amount sent was tripled by the experimenter. Receivers had to choose a portion of this tripled amount in multiples of $£ 0.50$ (U.S. $\$ 0.75$ ) to return to the sender.

The experimental sessions of the VE-setting were conducted entirely within SL, following an approach that reflected the PE-setting in as much detail as possible. However, some experimental procedures were adjusted to accommodate SL. Unlike scheduled sessions in the laboratory, we solicited participation by approaching online users in situ immediately before a particular experimental session in the following manner. Half an hour before a session, we used the search feature in the SL-interface to identify the currently busiest locations regarding number of avatars present. The experimenter then used an avatar to access these locations and to address groups of avatars present using public instant messaging (IM) with a standardised recruitment message in English stating our institutional affiliation and general information about the nature of the task, its duration and incentivisation. Whenever interested users responded, any additional questions were answered, and volunteers informed of the time and venue of the session. This process was repeated for some locations and avatar groups in each until the recruitment of the desired number of participants was complete. In this way, we recruited a total of 60 participants in SL, i.e. 30 senderreceiver pairs. Interested participants were teleported to our virtual experimental laboratory in a dedicated virtual building with controllable access rights and purpose-built furniture. In the briefing stage, participants were given virtual documents containing general information on experimental etiquette, anonymity, confidentiality and incentivisation. The experimenter communicated with participants using either public or private (i.e. one-to-one) IM. Once participants finished reading the briefing documents they were asked to occupy virtual cubicles designed to restrict vision and communication to prevent collusion between them. They were then given virtual documents containing the experimental instructions and a comprehension quiz. The task commenced after all participants completed the quiz correctly. Participants communicated their decisions to the lead experimenter and received feedback via private IM. In the payment stage of the experimental session, participants were paid earnings in L\$ on the spot using the SL payment transfer feature. Regarding experimental parameters, senders chose a division of a L\$1000 stake (U.S. \$4) in multiples of $\mathrm{L} \$ 1$ to be sent to the receiver. The amount sent was again tripled. Receivers chose a portion of this tripled amount in multiples of L\$1 to return to the sender. Also, participants were paid a show-up fee of L\$ 500 each. A typical experimental session in progress is shown in Figure 2.

Regarding exchange rate, our SL stake differs from that used in the physical setting. However, the lower hourly wage in SL means that participants' potential earnings are comparable in the two settings. According to the standard method for incentive-compatible experiments, participants' 
expected money earnings are calibrated in line with their particular opportunity cost of participation (e.g. Friedman \& Sunder, 1994). This is commonly estimated using the prevalent hourly wage for casual work. As a result, calibration may need to be adjusted to create equivalent compensation between different types of participant groups in a given experiment. For example, when participants from both developing and developed societies are compared within a single study, their respective experimental earnings are translated into cash at differential rates based on wage levels and their purchasing power at the local level (e.g. Henrich et al., 2004). We adopt a similar approach here. We estimated the opportunity cost of virtual participation using the prevalent rates for virtual labour within SL, sometimes referred to as farming, which is less than the opportunity cost in physical locations.

Finally, our EE-setting was designed to represent the salient features of e-commerce, and we employed a website environment for this purpose. Participants were given the address of a webpage located on our institution's webserver that contained experimental instructions and a comprehension quiz. Question and instruction wording, comprehension quiz questions and experimental incentives were identical to those used in VE. The webpage was designed plainly consisting of only text other than our institutional affiliation and logo at the top against white background. Successful completion of the quiz questions produced a second webpage with the experimental task. Upon completion of this task, the results were e-mailed to an address containing our institutional domain name.

The participant recruitment process was also conducted within SL and followed the protocol of the VE-setting in every detail. The reason is that our experimental results can only reveal the difference between the EE and VE-settings if they are the same in all other aspects, including composition and nature of the participant pool. As our treatment variable is the nature of the electronic interface, i.e. e-commerce versus v-commerce, any additional difference between the corresponding settings would have confounded its effect. All participants were informed to complete the task using a web browser within 12 hours of their recruitment and given a participation code. We completed the data collection for our desired number of senders before recruiting receivers. While all participants recruited as senders were directed to the same page, receivers were given individual webpages containing the amount sent by their randomly-assigned and anonymous senders previously recruited. All participants were paid out using SL's transfer feature once all participant decisions were received. Unsolicited participants directed to the webpages by people other than the experimenters did not have valid participation codes and their responses were not included. 


\section{Results}

The dataset our design generated consists of the behaviour we observed regarding trustingness (measured as \% of the stake sent by senders) and trustworthiness (\% of the received amount returned by receivers). Table I provides summary statistics. Out of our four hypotheses, two (H3 and H4) were supported fully and two (H1 and H2) partially by our data.

\section{Trustingness (H1 and H3)}

The first step in our analysis is to test for differences in trustingness between the treatments including the experiment conducted in the physical laboratory setting. We rely on previous literature that supports a meaningful comparison between virtual experimental and standard physical laboratory behaviour. In particular, Chesney et al. (2009) examined to what extent SL can be used as an experimental platform to generate results that may be compared with conventional laboratory studies. These authors experimented with a series of standard incentive-compatible tasks within SL using participants recruited entirely in this virtual world as well as in standard physical laboratories and found that both groups may be validly compared. They, and other studies such as Yee (2006), explored the demographics and social values of SL participants and concluded their profiles are sufficiently similar to those of standard participants to allow such comparisons.

As can be seen in Table I, the average amount sent is highest in the VE-setting (61\%), followed by PE (55\%) and EE (38\%). A Kruskal-Wallis test confirmed that these averages are significantly different $(p=0.0001)$. To ascertain which particular conditions differ from one another, we also report pairwise Mann-Whitney tests. As experimental data typically do not obey normality assumptions, non-parametric tests are often preferable (e.g. Davis \& Holt, 1993, p. 526). The resulting test statistics for the pairwise comparisons between the three environments are presented in Table II. The results of both tests suggest that there is significantly less trusting behaviour in the electronic trading environment compared to both the physical and virtual setting. Also, no difference between the latter two environments is in evidence. We interpret this as support for H3, but only partial support for H1.

\section{Trustworthiness (H2 and H4)}

We now turn to the analysis of trustworthiness regarding amounts returned by receivers. Table I suggests higher trustworthiness (38\%) in the VE-settings to both $\mathrm{EE}$ and PE, while there is a relatively small difference between the average of these (24\% and $27 \%$ respectively). Again, we conducted a Kruskal-Wallis test for differences in these averages, which was highly significant 
( $p=0.0001)$. To examine pairwise differences, Mann-Whitney tests were performed in a pairwise fashion and confirm higher trustworthiness in the virtual environment compared to the other two settings, which, in turn, exhibited no significant difference between them (Table III). However, as receivers' decisions are made under perfect information regarding the decision of senders, we also used a multivariate testing method as amounts returned potentially depend on the corresponding amounts sent by the sender. The equation was estimated using the ordinary least squares technique and produced the results reported in Table IV. We used the VE-data as a baseline and introduced dummies for the other two settings as independent variables along with the amount sent, which were regressed on amount returned. Again, the results offer support for H4, but only partial support for $\mathrm{H} 2$. The regression results show significant negative coefficients for $\mathrm{EE}$ and $\mathrm{PE}$, confirming that virtual world receivers returned more controlling for the amounts they were sent. However, the latter variable is insignificant, suggesting trustworthiness is not significantly explained by trustingness. This result is in line with some previous studies. In our dataset, we did not record the amount returned by receivers who were sent zero amounts, as they had no decision to make. If instead, we record zero returns for these cases, the amount sent becomes a significant predictor in the regression. Previous studies approach this issue in different ways, which may explain why some find amounts sent to be significant (e.g. Croson \& Buchan, 1999) while others do not (e.g. Berg et al., 1995; Willinger et al., 2003)

\section{Discussion}

The objective of this paper was to assess the role of v-commerce in promoting trust in computermediated transactions thereby responding to calls for more research into the role of context and trustee attributes (e.g. Bansal et al., 2016) and the stumbling blocks to firms adopting virtual worlds (Yoon \& George, 2013). This research also adds to the discussion of e- and v- commerce and, in particular, the deeper understanding of real benefits rather than hyped expectations (Wasko, Teigland, Leidner, \& Jarvenpaa, 2011).

Our research is unique in that it focuses on trust behaviour rather than attitudes. Our findings show that traders' trust anonymous others and that trust is returned. Further, trust differs significantly between web-based e-commerce and virtual environments. Both trustingness and trustworthiness are significantly higher in the virtual commerce setting compared with the static electronic setting. To the extent that our experimental design appropriately represented these settings, and that our chosen measures of trust are valid, we may conclude that v-commerce is capable of promoting trust in 
computer-mediated transactions to the equal of bricks-and-mortar settings, though we acknowledge that further research is warranted.

Based on participants' actions, no difference in trusting behaviour could be detected between the physical and virtual environments. However, there is a difference in trustworthiness, with participants' actions highlighting a greater degree of trustworthiness in the virtual setting compared with the physical setting. These findings are interesting as trustingness involves expectations of others' reciprocity. A sender may have ample reason to be more suspicious of an online trade with a faceless (anonymous) person. However, the representation by an avatar becomes the surrogate for physical interactions with the inherent trust that such an interaction would bring. Further, while trustworthiness is not characterised by the actions of another person, the greater returns made by virtual environment participants may also reflect the information richness generated by this medium, thus correcting the trust deficit.

We therefore find that trust is no less and sometimes greater in the virtual compared to the physical environment, contrary to our hypotheses $\mathrm{H} 2$ and $\mathrm{H} 4$ based on information richness theory. In this theory the physical environment is the most information rich and should harbour a greater capacity for mutual trust. Our result chimes however with that of Füllbrunn et al. (2011) who also find higher trustworthiness by SL-receivers than by those in their physical laboratory. While our experiment was not designed to uncover the reasons for this unexpected result, there are two possibilities from other studies. One is a reduction in social inhibition in virtual contexts that may temper trust in physical settings (Chesney, Coyne, Logan, \& Madden, 2009). Another is that virtual environments may be associated with cooperative social conventions that developed based on shared particular interests. For example, Becker and Mark (2002, p. 36) suggest virtual technologies may have an "integrative social effect, and [...] counteract the tendencies of fragmentation and individualization in modem societies". Further work in this direction is needed.

In comparison with a physical setting, the virtual space offers the same indicators for trust (trustingness) and greater potential for trustworthiness far more than the e-commerce or static online space. Thus, the virtual setting combines both the best features of a traditional brick-and-mortar retail environment with the economics of scale and global reach of e-commerce. Future research focussing on the physical, e- and v-commerce conditions across multiple service situations and communities would be worthwhile to aid deeper understanding of trust in different contexts and with different types of trades and traders. The implications for marketing practitioners and online businesses are significant. For example, e-businesses need to consider trust implications for their commerce space and look to strategies and tactics to promote both dimensions of trust. In particular, e-tailers may exploit virtual world technology as a surrogate for the trust indicators inherent within brick-and- 
mortar environments. One possibility is to clearly establish information richness within virtual worlds, possibly through the graphical representation of users by avatars, and the options to display information about them (e.g. their social activities), in order to simulate aspects of physical, social networking and recreate an artificial but acceptable substitute for face-to-face interactions.

Additionally, trust may be increased through the use of a virtual world element as part of the online interface where customers experience information richness in the form of employees' avatars as well as other customers' avatars. SL successfully engenders information richness (Nowak \& Biocca, 2003) in a far advanced manner than simply adding a social touch (e.g. personalised welcome message or photographs of smiling people) to the presence of a static website (Gefen \& Straub, 2004). Our results show the potential for commerce within virtual worlds to bridge the gap between physical and electronic trades by exploiting the advantages of both. Information richness is critical as it represents the indicators of trust to prospective and current customers thereby reducing risk and uncertainty in trial behaviour and reinforcing positive aspects associated with repeat purchase behaviour (brand and community building, referrals and loyalty).

While rapid technological change presents constant challenges for marketing practitioners, it also offers opportunities for the development and implementation of new strategies. For example, simulations of physical social networks may change frequently in real-world applications, such changes can be replicated in the virtual commerce world. Just as a customer may prefer a particular shop assistant at their regular shop, online customers could be given the option to select a preferred avatar (or virtual shop assistant). Online customers could also be given options to further engage with avatars, such as referring them to their friends, customising them to suit preferences (and to build upon trust through appearance or manner), assigning personal shopping avatars to provide advice on purchases for self or others or arrange online group shopping excursions and shopping clubs.

The last decade has seen immense growth in the number of virtual worlds, their users and consequently, the market size of virtual economies. For example, as of 2014, among the over 1 million active SL users alone, total in-world transactions amounted to U.S. \$ 3.2 billion (LindenLab, 2013). Besides SL, virtual worlds such as World of Warcraft and Entropia Universe have also seen significant growth in their user base and economies (Nazir \& Lui, 2016). Such growth has attracted real-world companies and individual entrepreneurs to seek new commercial opportunities in virtual economies. Virtual supermarkets are expected in the near future (Khan \& Brouwer, 2016). Apart from commerce, virtual worlds will also have a place in the future for entertainment and education. Within education, for instance, a large number of established institutions have a presence within virtual worlds including University of Illinois, Carnegie Mellon University and Harvard University 
(Downey, 2014). Our findings contribute to the process of engendering trust to oil the wheels of such virtual economies and activities.

Finally, for service companies, the implications for trust on behaviour highlights an opportunity to stimulate consumption and reduce purchase or trial uncertainty, and the need to carefully craft a servicescape which leverages information richness and trust signals.

Our conclusions have to be somewhat qualified to account for the general limitations of our study. First, for the reasons explained previously, our approach was not to study trust in naturalistic settings or as attitudes or intentions, but as observed behaviour in abstract, interactive and incentivised decision tasks between players of an economic game. While this feature of our study is a novel contribution to the trust in e-commerce literature it may be argued that the trust game constitutes too great an abstraction from realistic commercial situations to generate reliable conclusions regarding richer occurrences of trust in naturalistic scenarios. On the other hand, irrespective of the abstractness of the task, trust game behaviour does measure some aspect of trust, especially to the extent that it is incentivised. Also, it is exactly this abstraction that allows us to distil the essence of trust behaviour without other confounding influences (Camerer 2003).

Secondly, one may be suspicious of the specific validity and reliability of experimental results generated in a virtual environment lacking the control of physical laboratories. However, there is support for SL-experimentation which has been shown to be capable of reproducing the results of experiments conducted in standard, physical laboratory experiments across a number of experimental tasks (Chesney, Chuah, et al., 2009). Moreover, the study of a virtual world, and in particular the collection of behavioural data there, represents a novel feature of our work in this paper.

Similar to other experimental studies (e.g. Henrich, 2000), our sample size is relatively modest when compared to studies based on surveys which reflects experimental economy and logistical challenges. However, the analytical techniques we use (especially non-parametric techniques) account for number of observations and the potential for non-normal distribution of data and still generated significant results. Further studies to replicate our findings would be a welcome addition to the literature.

It should also be noted that our approach to varying information richness as an experimental treatment effect involves presence between experimenter and participant, not between participants. Participants' particular co-players are absent and strictly anonymous in all our settings. Further research could explore the relational nature of trust in more detail.

While our results are suggestive of the efficacy of the artificially-created information richness inherent in virtual worlds, the study was not designed to uncover the underlying reasons for them. The most likely source of virtual trust may indeed lie in the way it is designed to simulate aspects of 
the physical world, including human physical signals and non-verbal communication such as gestures and body language. Future work in this direction seems warranted. 
Table I Summary statistics of trustingness (\% sent) and trustworthiness ( $\%$ returned)

\begin{tabular}{|c|c|c|c|c|c|c|}
\hline & Environn & ent & & & & \\
\hline & PE & & VE & & $\mathbf{E E}$ & \\
\hline Behaviour & $\%$ sent & $\begin{array}{l}\% \\
\text { returned }\end{array}$ & $\%$ sent & $\begin{array}{l}\% \\
\text { returned }\end{array}$ & $\% \operatorname{sent}$ & \% returned \\
\hline Mean & 55.47 & 26.87 & 61.17 & 38.49 & 38.48 & 24.47 \\
\hline $\begin{array}{l}\text { Mode } \\
\text { (percent) }\end{array}$ & $\begin{array}{l}50(22.9) \\
100 \\
(22.9)\end{array}$ & $\begin{array}{l}33.33 \\
(28.2)\end{array}$ & $\begin{array}{l}100 \\
(36.7)\end{array}$ & $50(40.7)$ & $\begin{array}{l}30 \\
(15.6) \\
50 \\
(15.6)\end{array}$ & $0(25)$ \\
\hline Std. Dev. & 35.53 & 18.86 & 37.09 & 21.34 & 29.1 & 26.06 \\
\hline Observations & 48 & 39 & 30 & 27 & 32 & 32 \\
\hline
\end{tabular}

Table II Differences in trustingness (\% sent)

\begin{tabular}{|l|l|l|l|}
\hline Environment & PE & VE & EE \\
\hline PE & & $t=-0.68(0.5)$ & $t=2.25\left(0.027^{* *}\right)$ \\
& & $U=654.00(0.49)$ & $U=544.50\left(0.027^{* *}\right)$ \\
\hline VE & & & $t=2.67\left(0.01^{* *}\right)$ \\
& & & $U=313.50\left(0.018^{* *}\right)$ \\
\hline
\end{tabular}

Notes: Significance tests reported in terms of $t$-statistics and Mann-Whitney $U$. Two-tailed $p$-values in parentheses. $* * p<0.05 ; * * * p<0.01$.

Table III Differences in trustworthiness (\% returned)

\begin{tabular}{|l|l|l|l|}
\hline Environment & PE & VE & EE \\
\hline PE & & $\begin{array}{l}t=-2.33\left(0.02^{* *}\right) \\
U=316.00\left(0.006^{* * *}\right)\end{array}$ & $\begin{array}{l}t=0.45(0.66) \\
U=509.00(0.18)\end{array}$ \\
\hline VE & & $\begin{array}{l}t=2.23(0.03 * *) \\
U=230.50\left(0.002^{* * *}\right)\end{array}$ \\
\hline
\end{tabular}

Notes: Significance tests reported in terms of $t$-statistic and Mann-Whitney $U$. Two-tailed p-values in parentheses. $* * p<0.05 ; * * * p<0.01$. 
Table IV Regression results for trustworthiness (\% returned)

\begin{tabular}{|l|l|l|}
\hline Independent Variable & Coefficient & $\boldsymbol{p}$-value \\
\hline Constant & 38.12 & $0.000^{* * *}$ \\
\hline Amount sent & 0.005 & 0.95 \\
\hline Physical $^{\mathrm{a}}$ & -11.62 & $0.039^{* *}$ \\
\hline Electronic $^{\mathrm{b}}$ & -13.88 & $0.026^{* *}$ \\
\hline$F$ & 2.2 & 0.093 \\
\hline Adjusted $R^{2}$ & 0.036 & \\
\hline Observations & 98 & \\
\hline
\end{tabular}

Notes: ${ }^{\text {a,b }}$ dummies for physical and electronic environments respectively. ${ }^{* *} p<0.05 ; * * * p<0.01$. 
Figure 1 Second Life avatars in a shop

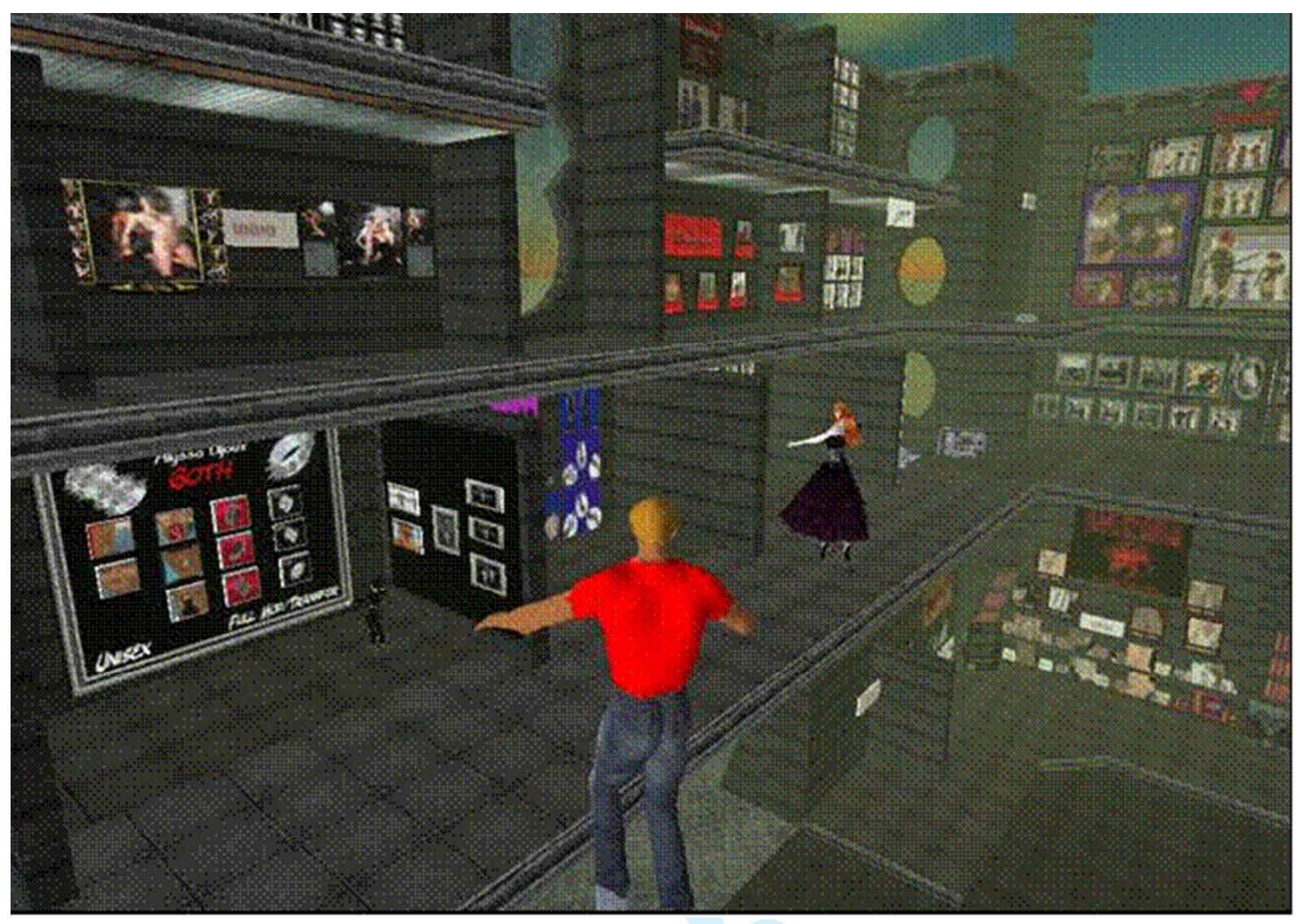


Figure 2 An experiment in Second Life

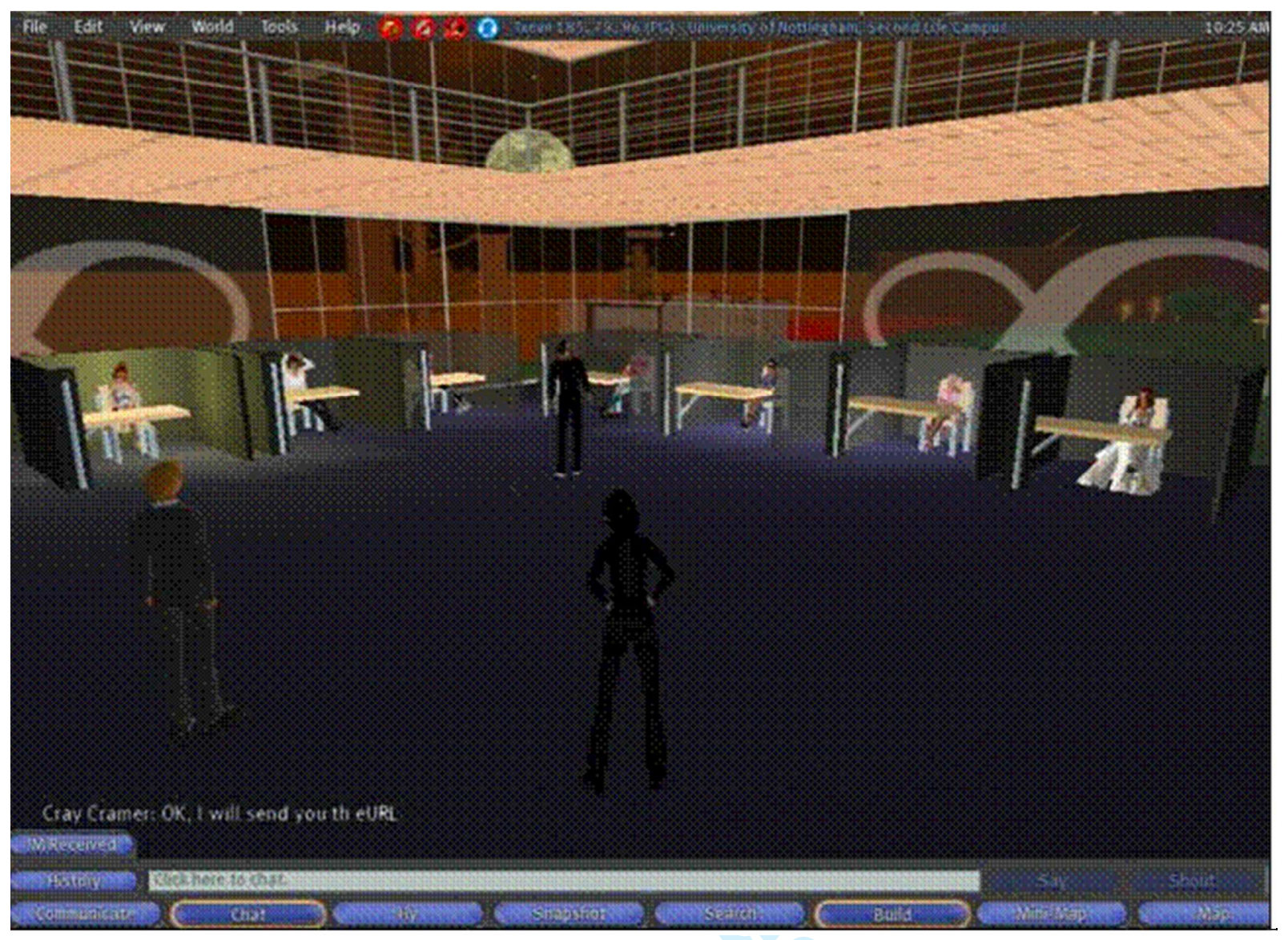


References

Agarwal, J., \& Wu, T. (2015). Factors Influencing Growth Potential of E $\square$ Commerce in Emerging Economies: An Institution $\square$ Based $\mathrm{N} \square$ OLI Framework and Research Propositions. Thunderbird International Business Review, 57(3), 197-215.

Argyle, M., \& Dean, J. (1965). Eye contact, distance and affiliation. Sociometry, 28(3), 289-304.

Atlas, S., \& Putterman, L. (2011). Trust among the avatars: A virtual world experiment, with and without textual and visual cues. Southern Economic Journal, 78(1), 63-86.

Bainbridge, W. S. (2007). The Scientific Research Potential of Virtual Worlds. Science, 317(5837), 472.

Bansal, G., Zahedi, F. M., \& Gefen, D. (2016). Do context and personality matter? Trust and privacy concerns in disclosing private information online. Information \& Management, 53(1), 1-21.

Barnes, S. J., Mattsson, J., \& Hartley, N. (2015). Assessing the value of real-life brands in Virtual Worlds. Technological Forecasting and Social Change, 92, 12-24

Barrett, M., Davidson, E., Prabhu, J., \& Vargo, S. L. (2015). Service innovation in the digital age: key contributions and future directions. MIS Quarterly, 39(1), 135-154.

Bauer, H. H., Albrecht, C.-M., Neumann, M. M., \& Haber, T. E. (2015). Enhancing Customer Trust in E-Commerce Through Web Portals Revolution in Marketing: Market Driving Changes (pp. 57-61): Springer.

Beatty, P., Reay, I., Dick, S., \& Miller, J. (2011). Consumer trust in e-commerce web sites: A metastudy. ACM Computing Surveys (CSUR), 43(3), 1-46.

Becker, B., \& Mark, G. (2002). Social conventions in computermediated communication: A comparison of three online shared virtual environments. In R. Schroeder (Ed.), The Social Life of Avatars: Presence and Interaction in Shared Virtual Environments (pp. 19-39 ): Springer.

Beldad, A., De Jong, M., \& Steehouder, M. (2010). How shall I trust the faceless and the intangible? A literature review on the antecedents of online trust. Computers in Human Behavior, 26(5), 857-869.

Bente, G., Ruggenberg, S., Kramer, N. C., \& Eschenburg, F. (2008). Avatar-Mediated Networking: Increasing Social Presence and Interpersonal Trust in Net-Based Collaborations. Human Communication Research, 34(2), 287-318.

Berg, J., Dickhaut, J., \& McCabe, K. (1995). Trust, Reciprocity, and Social History. Games and Economic Behavior, 10(1), 122-142. 
Berry, L. L. (2000). Cultivating service brand equity. Journal of the Academy of Marketing Science, $28(1), 128-137$.

Bertrand, M., \& Mullainathan, S. (2001). Do people mean what they say? Implications for subjective survey data. AMERICAN ECONOMIC REVIEW, 91(2), 67-72

Biocca, F., Burgoon, J., Harms, C., \& Stoner, M. (2001). Criteria and scope conditions for a theory and measure of social presence. Paper presented at the Presence 4th Annual International Workshop, Philadelphia, PA.

Blascovich, J., Loomis, J., Beall, A. C., Swinth, K. R., Hoyt, C. L., \& Bailenson, J. N. (2002). Immersive virtual environment technology as a methodological tool for social psychology. Psychological Inquiry, 13(2), 103-124.

Blau, P. M. (1964). Power and Exchange in Social Life: New York: Wiley.

Bolle, F. (1998). Rewarding trust: An experimental study. Theory and Decision, 45(1), 83-98

Butler, J. K. (1991). Toward Understanding and Measuring Conditions of Trust: Evolution of a Conditions of Trust Inventory. Journal of Management, 17(3), 643-663.

Camerer, C. (2003). Behavioral Game Theory: Experiments in Strategic Interaction. Princeton: Princeton University Press

Camerer, C., \& Fehr, E. (2004). Measuring social norms and preferences using experimental games: a guide for social scientists. In J. Henrich, R. Boyd, S. Bowles, C. Camerer, E. Fehr, \& H. Gintis (Eds.), Foundations of Human Sociality: Economic Experiments and Ethnographic Evidence from Fifteen Small-Scale Societies. Oxford: Oxford University Press.

Carlson, J. R., \& Zmud, R. W. (1999). Channel expansion theory and the experiential nature of media richness perceptions. Academy of Management Journal, 42(2), 153-170.

Castelfranchi, C., \& Tan, Y.-H. (2001). The role of trust and deception in virtual societies. Paper presented at the Proceedings of the 34th Annual Hawaii Int. Conference on System Sciences, Wailea, Hawaii, Jan.

Castronova, E. (2008). Synthetic worlds: The business and culture of online games: University of Chicago press.

Chambers, C. (2011). How virtual are virtual economies? An exploration into the legal, social and economic nature of virtual world economies. Computer Law \& Security Review, 27(4), 377384.

Chandon, P., Morwitz, V. G., \& Reinartz, W. J. (2005). Do intentions really predict behavior? Selfgenerated validity effects in survey research. Journal of Marketing, 69(2), 1-14

Chesney, T., Chuah, S. H., \& Hoffmann, R. (2009). Virtual World Experimentation: An Exploratory Study. Journal of Economic Behavior and Organization, 72(1), 618-635. 
Chesney, T., Coyne, I., Logan, B., \& Madden, N. (2009). Griefing in virtual worlds: causes, casualties and coping strategies. Information Systems Journal, 19(6), 525-548

Coyle, J. R., \& Thorson, E. (2001). The effects of progressive levels of interactivity and vividness in web marketing sites. Journal of Advertising, 30(3), 65-77

Croson, R. (2005). The method of experimental economics. International Negotiation, 10(1), 131148

Croson, R., \& Buchan, N. (1999). Gender and Culture: International Experimental Evidence from Trust Games. American Economic Review, 89(2), 386-391.

Cunningham, J. A., Rahman, I. A., Lautenschlager, S., Rayfield, E. J., \& Donoghue, P. C. (2014). A virtual world of paleontology. Trends in ecology \& evolution, 29(6), 347-357.

Cyr, D., Hassanein, K., Head, M., \& Ivanov, A. (2007). The role of social presence in establishing loyalty in e-Service environments. Interacting with Computers, 19(1), 43-56.

Daft, R. L. (2013). Information richness theory. In E. H. Kessler (Ed.), Encyclopedia of Management Theory: Sage Publications.

Daft, R. L., \& Lengel, R. H. (1986). Organizational information requirements, media richness and structural design. Management Science, 32(5), 554-571.

Daft, R. L., Lengel, R. H., \& Trevino, L. K. (1987). Message equivocality, media selection, and manager performance: Implications for information systems. MIS Quarterly, 11(3), 355-366.

Davis, D. D., \& Holt, C. A. (1993). Experimental Economics. Princeton: Princeton University Press.

Doney, P. M., \& Cannon, J. P. (1997). An examination of the nature of trust in buyer-seller relationships. Journal of Marketing, 35-51

Downey, S. (2014). History of the (virtual) worlds. The Journal of Technology Studies, 40(2), 54-66.

Duffy, J. (2011). Trust in second life. Southern Economic Journal, 78(1), 53-62

Dwyer, F. R., Schurr, P. H., \& Oh, S. (1987). Developing buyer-seller relationships. Journal of Marketing, 51(2), 11-27.

El-Shinnawy, M., \& Markus, M. L. (1998). Acceptance of communication media in organizations: richness or features? Professional Communication, IEEE Transactions on, 41(4), 242-253.

Fang, Y., Qureshi, I., Sun, H., McCole, P., Ramsey, E., \& Lim, K. H. (2014). Trust, Satisfaction, and Online Repurchase Intention: The Moderating Role of Perceived Effectiveness of ECommerce Institutional Mechanisms. MIS Quarterly, 38(2), 407-427

Friedman, D., \& Sunder, S. (1994). Experimental methods: A primer for economists: Cambridge university press.

Fukuyama, F. (1995). Trust: The Social Virtues and the Creation of Prosperity. New York: Free Press. 
Füllbrunn, S., Richwien, K., \& Sadrieh, A. (2011). Trust and trustworthiness in anonymous virtual worlds. Journal of Media Economics, 24(1), 48-63

Gefen, D., Benbasat, I., \& Pavlou, P. (2008). A Research Agenda for Trust in Online Environments. Journal of Management Information Systems, 24(4), 275-286.

Gefen, D., \& Silver, M. (1999). Lessons learned from the successful adoption of an ERP system. Paper presented at the Fifth International Conferences of the Decision Sciences Institute, Athens, Greece.

Gefen, D., \& Straub, D. W. (2004). Consumer trust in B2C e-Commerce and the importance of social presence: experiments in e-Products and e-Services. Omega, 32(6), 407-424.

Giffin, K. (1967). The contribution of studies of source credibility to a theory of interpersonal trust in the communication process. Psychological Bulletin, 68(2), 104-120.

Glaeser, E. L., Laibson, D. I., Scheinkman, J. A., \& Soutter, C. L. (2000). Measuring Trust. Quarterly Journal of Economics, 115(3), 811-846.

Grabner-Kräuter, S., \& Bitter, S. (2013). Trust in online social networks: A multifaceted perspective. Forum for Social Economics, 44(1), 48-68. doi:10.1080/07360932.2013.781517

Grabner-Kräuter, S., \& Kaluscha, E. A. (2003). Empirical research in on-line trust: a review and critical assessment. International Journal of Human-Computer Studies, 58(6), 783-812.

Greiner, B., Caravella, M., \& Roth, A. E. (2014). Is avatar-to-avatar communication as effective as face-to-face communication? An Ultimatum Game experiment in First and Second Life. Journal of Economic Behavior \& Organization, 108, 374-382

Henrich, J. (2000). Does culture matter in economic behavior? Ultimatum game bargaining among the Machiguenga of the Peruvian Amazon. AMERICAN ECONOMIC REVIEW, 90(4), 973979

Henrich, J., Boyd, R., Bowles, S., Camerer, C., Fehr, E., \& Gintis, H. (Eds.). (2004). Foundations of Human Sociality-Economic Experiments and Ethnographic: Evidence From Fifteen SmallScale Societies. Oxford: Oxford University Press.

Holm, H. J., \& Danielson, A. (2005). Tropic Trust Versus Nordic Trust: Experimental Evidence From Tanzania And Sweden. The Economic Journal, 115(503), 505-532.

Hupcey, J. E., Penrod, J., Morse, J., \& Mitcham, C. (2001). An exploration and advancement of the concept of trust. Journal ofAdvanced Nursing, 36(2), 282-293. doi:doi:10.1046/j.13652648.2001.01970.x

Inglehart, R. (1997). Modernization and Postmodernization: Cultural, Economic, and Political Change in 43 Societies. Princeton, N.J.: Princeton University Press.

Johnson, C., Hult, P., \& McGowan, B. (2008). Web buyers and their expectations grow up: 
Experienced web buyers are becoming the new mainstream. Retrieved from http://www.forrester.com/Research/PDF/0,5110,45462,00.pdf

Johnson, N. D., \& Mislin, A. A. (2011). Trust games: A meta-analysis. Journal of Economic Psychology, 32(5), 865-889

Kelton, K., Fleischmann, K. R., \& Wallace, W. A. (2008). Trust in digital information. Journal of the American Society for Information Science and Technology, 59(3), 363-374

Keser, C. (2002). Trust and Reputation Building in E-commerce: CIRANO.

Khan, V., \& Brouwer, R. (2016). The relation between customer types in a real supermarket compared to a virtual supermarket. Journal For Virtual Worlds Research, 9(1), 1-16.

Knowles, I., Castronova, E., \& Ross, T. (2015a). 11. Video games, virtual worlds and economics. Handbook on the Economics of the Media, 237-259.

Knowles, I., Castronova, E., \& Ross, T. (2015b). Virtual Economies: Origins and Issues. The International Encyclopedia of Digital Communication and Society.

Kock, N. (2004). The psychobiological model: Towards a new theory of computer-mediated communication based on Darwinian evolution. Organization Science, 15(3), 327-348

Komiak, S. X., \& Benbasat, I. (2004). Understanding Customer Trust in Agent-Mediated Electronic Commerce, Web-Mediated Electronic Commerce, and Traditional Commerce. Information Technology and Management, 5(1), 181-207.

Kumar, N. (1996). The Power of Trust in Manufacturer-Retailer Relationships. Harvard Business Review, 74(Nov-Dec), 92-110.

LindenLab. (2013). Infographic: 10 years of Second Life [Press release]. Retrieved from http://www.lindenlab.com/releases/infographic-10-years-of-second-life

Lomanowska, A. M., \& Guitton, M. J. (2014). My avatar is pregnant! Representation of pregnancy, birth, and maternity in a virtual world. Computers in Human Behavior, 31, 322-331.

Lu, Y., Kim, Y., Dou, X. Y., \& Kumar, S. (2014). Promote physical activity among college students: Using media richness and interactivity in web design. Computers in Human Behavior, 41, 4050.

Luhmann, N. (1979). Trust and Power. Chichester: John Wiley \& Sons.

Mayer, R. C., Davis, J. H., \& Schoorman, F. D. (1995). An integrative model of organizational trust. Academy of Management Review, 20(3), 709-734.

McKnight, D. H., Choudhury, V., \& Kacmar, C. (2003). Developing and Validating Trust Measures for e-Commerce: An Integrative Typology. Information Systems Research, 13(3), 334-359.

Mutz, D. C. (2005). Social Trust and E-Commerce Experimental Evidence for the Effects of Social Trust on Individuals' Economic Behavior. Public Opinion Quarterly, 69(3), 393-416. 
Nazir, M., \& Lui, C. (2016). A brief history of virtual economy. Journal of Virtual Worlds Research, 9(1), 1-24.

Noteborn, G., Dailey-Hebert, A., Carbonell, K. B., \& Gijselaers, W. (2014). Essential knowledge for academic performance: Educating in the virtual world to promote active learning. Teaching and Teacher Education, 37, 217-234

Nowak, K. L., \& Biocca, F. (2003). The Effect of the Agency and Anthropomorphism on Users' Sense of Telepresence, Copresence, and Social Presence in Virtual Environments. Presence: Teleoperators \& Virtual Environments, 12(5), 481-494.

Ostrom, A. L., Parasuraman, A., Bowen, D. E., Patrício, L., Voss, C. A., \& Lemon, K. (2015). Service research priorities in a rapidly changing context. Journal of Service Research, 18(2), 127-159

Postmes, T., Spears, R., Sakhel, K., \& de Groot, D. (2001). Social Influence in Computer-Mediated Communication: The Effects of Anonymity on Group Behavior. Personality and Social Psychology Bulletin, 27, 1243-1254.

Prentice, S. (2007). Virtual worlds: To Second Life and beyond. Sydney, Australia: Gartner Symposium/ITxpo.

Quigley, J. F. (2015). Consumer behavior in digital markets. (Master of Science), Southern Illinois University Carbondale, Southern Illinois, USA.

Rockmann, K. W., \& Northcraft, G. B. (2008). To be or not to be trusted: The influence of media richness on defection and deception. Organizational Behavior and Human Decision Processes, 107(2), 106-122.

Roth, A. E. (1988). Laboratory Experiments in Economics: A Methodological Overview. Economic Journal, 98(393), 974-1031.

Rotter, J. B. (1971). Generalized expectancies for interpersonal trust. American Psychologist, 26(5), 443-452.

Rotter, J. B. (1980). Interpersonal Trust, Trustworthiness, and Gullibility. American Psychologist, $35(1), 1-7$.

San Martín, S., \& Camarero, C. (2005). Consumer reactions to firm signals in asymmetric relationships. Journal of Service Research, 8(1), 79-97

Schlichter, B. R., \& Rose, J. (2013). Trust dynamics in a large system implementation: six theoretical propositions. European Journal of Information Systems, 22(4), 455-474

Shin, D.-H., \& Shin, Y.-J. (2011). Consumers' trust in virtual mall shopping: The role of social presence and perceived security. International Journal of Human-Computer Interaction, 27(5), 450-475 
Simon, S. J., \& Peppas, S. C. (2004). An examination of media richness theory in product Web site design: an empirical study. info, 6(4), 270-281.

Toufaily, E., Souiden, N., \& Ladhari, R. (2013). Consumer trust toward retail websites: Comparison between pure click and click-and-brick retailers. Journal of Retailing and Consumer Services, 20(6), 538-548

Verhallen, T. M. M., \& Pieters, R. G. M. (1984). Attitude theory and behavioral costs. Journal of Economic Psychology, 5(3), 223-249.

Wasko, M., Teigland, R., Leidner, D., \& Jarvenpaa, S. (2011). Stepping into the internet: New ventures in virtual worlds. MIS Quarterly, 35(3), 645-652

Waytz, A., Heafner, J., \& Epley, N. (2014). The mind in the machine: Anthropomorphism increases trust in an autonomous vehicle. Journal of Experimental Social Psychology, 52, 113-117.

Willinger, M., Keser, C., Lohmann, C., \& Usunier, J. C. (2003). A comparison of trust and reciprocity between France and Germany: Experimental investigation based on the investment game. Journal of Economic Psychology, 24(4), 447-466.

Yee, N. (2006). The demographics, motivations, and derived experiences of users of massively multiuser online graphical environments. Presence, 15(3), 309-329

Yoon, T. E., \& George, J. F. (2013). Why aren’t organizations adopting virtual worlds? Computers in Human Behavior, 29(3), 772-790.

Zhang, X., Ordóñez de Pablos, P., Wang, X., Wang, W., Sun, Y., \& She, J. (2014). Understanding the users' continuous adoption of 3D social virtual world in China: A comparative case study. Computers in Human Behavior, 35, 578-585. 